\title{
28 Resarach Soure \\ Validation And Psychometric Properties of The Trognon \& Richard ADHD Questionnaire 10-Items For Adults In A French Sample
}

Arthur Trognon ( $\nabla$ arthur.trognon-bertoni@univ-lorraine.fr)

University of Lorraine

Manon Richard

University of Lorraine

\section{Research Article}

Keywords: ADHD, diagnosis, machine-learning, clinical scale, psychometrics, adult

Posted Date: February 7th, 2022

DOI: https://doi.org/10.21203/rs.3.rs-1305661/v1

License: (c) (1) This work is licensed under a Creative Commons Attribution 4.0 International License.

Read Full License 


\section{Abstract}

Background: ADHD is classically seen as a disease of children, although it persists in one out of two cases in adults. The diagnosis is based on a long and multidisciplinary process, involving different health professionals, leading to an under-diagnosis of adult ADHD individuals. We therefore present a psychometric screening scale for the identification of adult ADHD, in order to serve as an aid in the decision whether or not to engage in a diagnostic process.

Method: We designed the scale from the DSM- 5 and administered it to $n=110$ control individuals and $\mathrm{n}=110$ ADHD individuals. The number of items was reduced using regression techniques. We then performed factor analyses and a machine-learning assessment of the predictive power of the scale.

Results: Internal consistency coefficients were calculated satisfactorily for TRAQ10, with Cronbach's alpha measured at .9. The 3-factor model tested was confirmed, with standardized factor loadings greater than .53 for all items. Finally, analysis by machine learning showed that a GNB-type classification algorithm could identify the subject's group appartenance with an high average precision of .88 based only on the participant's responses on the scale.

Conclusions: The scale showed sufficient performance for its use in clinical routine. It could thus help to reduce the time required to diagnose ADHD in adults. Similarly, it could be used in research for screening purposes.

\section{Introduction}

ADHD is a multifactorial disorder presenting a heterogeneous psychopathological profile with heterogeneous neurocognitive deficits, but with core symptoms implicating function of the frontalsubcortical-cerebellar pathways that control attention, saliency, inhibitory control and response to reward $^{1}$. This condition is classically looked as one of the most common childhood disease, but recent longitudinal studies accumulated evidences that ADHD persists into adulthood, with varying displayed characteristics among age ${ }^{2-4}$. In fact, while about $5 \%$ of children and adolescents are affected in the general population, this condition may persist in adulthood for at least half of these patients ${ }^{5}$. Several authors pointed out that this classical looking at ADHD has led to an underdiagnosis and undertreatment of adult $\mathrm{ADHD}^{6}$, because some professionals still express the fear of "treating a non-existent disease" while others are unsure about the diagnosis and the appropriate treatment of ADHD in adult mental health. These issues has led to strong international efforts to share information to clinicien, for example in the rework of the widely-used Diagnostic and Statistical Manual of Mental Disorders - Fifth Edition ${ }^{8}$, which highlighted the possible differential expression of ADHD throughout the patient's lifetime, and the European consensus statement on diagnosis and treatment of adult $\mathrm{ADHD}^{4}$, in order to facilitate the identification and the treatment of ADHD. 
Actually, the recognition of the importance of diagnosing and treating ADHD is growing, as it was recently shown that there is a higher prevalence of ADHD in some clinical populations such as addictive, forensic, and personality disorder patients ${ }^{9}$. In fact, in a general manner, ADHD was massively reported as a condition impairing individuals interaction with his environment, increasing the risk of the occurrence of harmful events such as injuries ${ }^{10}$, traffic accidents ${ }^{11}$, and substance abuse ${ }^{12}$, leading to increased healthcare utilization ${ }^{2}$, unemployment ${ }^{13}$, and suicide ${ }^{14}$. These observations pointed out the particular importance of screening within this high-risk populations.

However, ADHD is usually diagnosed through a time-consuming multidisciplinary evaluation process, including at least the visit to behavioral specialists such as psychologists and to mental functioning specialists such as neurologists or psychiatrists, increasing the diagnostic process duration which can extend over long periods of time. With the increasing need of screening, this led to the creation of psychometric auto-administered scales, such as the well-known Adult ADHD Self-Report Scale ${ }^{15}$, which was recently updated to the DSM-5 ${ }^{16}$, in order to shorten the diagnostic process. However, this work concerns a scale that has not been validated in French and thus cannot benefit to the French ADHD population and its need in terms of screening.

In order to meet this demand, we have developed an adult ADHD screening scale based on the DSM-5. We generated 45 preliminary items based on the DSM- 5 criteria, and administered this scale to 110 ADHD subjects and 110 controls. We then statistically selected the most discriminating items in regard to the presence or absence of the subject's clinical condition. We then analyzed its psychometric properties, in terms of internal consistency, as well as factor structure, under the prism of a 3-factor model accounting for the behavioral expression of cognitive symptomatology (i.e., attention; inhibition/impulsivity; and working memory). Finally, we examined the predictive power of the scale using a machine-learning approach, relying on operational metrics such as sensitivity, specificity, and predictive values.

\section{Material And Method}

\section{Subjects}

Two hundred and twenty not preselected adults (mean age $=27.8$ years, $S D=9.2 ; A D H D n=110$; controls $=110)$ from the general French population participated in this study. Full measures were available for all subjects.

All participants received detailed information about the study purpose and objectives, and provided online informed consent to participate in the study. All procedures were conducted in accordance with the Declaration of Helsinki and the study protocol was approved by the Institutional Review Board Commission Nationale de l'Informatique et des Libertés (registration n²224719v0).

\section{Scale development : item generation and selection}


Items were developed from the widely-used Diagnostic and Statistical Manual - Fifth Edition ${ }^{8}$. For each criterion, we generated 1 to 3 items (depending on whether an item overlapped several criteria), with the objective of bringing out a personal representation on which the participant could make a judgment on the concordance or otherwise between the induced representation and the subject's subjective feelings. 45 items were thus generated for the preliminary version of the scale. We selected a 6-points Osgoodstyle scale, ranging from 1 "Don't look like me at all." to 6 "Looks just like me.", in order to avoid neutrality bias, even if its existence is still currently debated ${ }^{17,18}$.

We then conducted a reiterative stepwise top-down regression multiple linear regression (diagnosis item $_{\mathrm{i}}$ ) in order to elucidate the factors by which the dependent variable was about to be influenced and for selecting discriminating items to highlight the diagnosis of ADHD. Then, we constructed our factor model on the remaining items relatively to the behavioural expression of the cognitive symptomatology of ADHD on three domains: attention, inhibition/impulsivity, and working memory deficits, accordingly to Butzbach et al., $2019^{19}$.

\section{Internal consistency and reliability}

Internal consistency and reliability of the remaining items was examined by Cronbach's alpha.

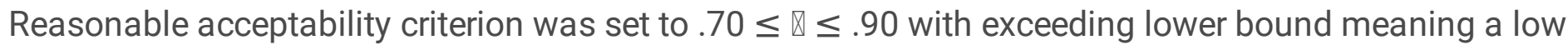
reliability, and exceeding higher bound meaning too many similar items, decreasing the scale's true reliability 20,21

\section{Factor structure}

In order to test our 3-factors model for TRAQ10 and assess construct validity, we conducted a confirmatory factor analysis. Generalized least squares method was performed in order to test the fit capability of the factor structure. Model fit was assessed using the following fit indices : we used the $\chi 2$ test statistic for absolute fit ; the comparative fit index (CFI) and Tucker-Lewis Index (TLI) for fit relative to a null model ${ }^{22,23}$; the Standardized Root Mean Square Residual $\left(\mathrm{SRMR}^{24}\right)$ and the Root Mean Square Error of Approximation (RMSEA ${ }^{25}$ ) for overall fit. Accordingly to Hu \& Bentler (1999) ${ }^{26}$, we assumed than our 3-factors model fit well if CFI>.95; TLI>.95; RMSEA $<.06$ and SRMR<.08. All statistical analyses were coded in R with Lavaan library and interpreted in RStudio v1.0.143.

\section{Evaluation of the scale's discriminating power}

To evaluate the scale's ability to capture the ADHD diagnosis, we chose to base our analysis on a machine-learning model. In our case (i.e. the prediction of a categorical data from a data matrix), the machine-learning approach consists in training a classifier on a subset and testing it on an independent subset. Then, the cross-validation procedure tests all possible partitioning (i.e. folds) of the data, always keeping the training set and the test set separate. Given the linear nature of the data and the likely presence of outliers, we selected a Gaussian Naive Bayes algorithm, which uses the pooled variance to make its probabilistic decision ${ }^{27}$. All machine-learning algorithms were coded and interpreted in Python. 


\section{Results}

\section{Multiple linear regression}

Multiple linear regression analysis (stepwise elimination procedure) using diagnosis as the dependent variable and patients' scores on TRAQ10 as independent variables (diagnosis item $\mathrm{m}_{\mathrm{i}}$ ) revealed that ADHD makes significatively vary scores on $\operatorname{TRAQ}_{1}[\beta=.047, p=.02], \operatorname{TRAQ}_{2}[\beta=.065, p=.007], \operatorname{TRAQ}_{3}[\beta=.034$, $p=.03], \operatorname{TRAQ}_{4}[\beta=-.06, p=.02], \operatorname{TRAQ}_{5}[\beta=-.05, p=.001], \operatorname{TRAQ}_{6}[\beta=-.08, p<.001], \mathrm{TRAQ}_{7}[\beta=-.003, p=.01]$,

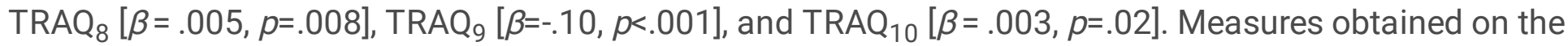
10 remaining items out of the 45 was $\left[F_{(10,209)}=38.8, p<.001, \mathrm{R}^{2}=.6499\right.$ and $\mathrm{R}^{2}$ adjusted $\left.=.6331\right]$.

\section{Internal consistency and reliability}

Table 1

Internal consistency and reliability

of the TRAQ10 if an item is dropped.

\begin{tabular}{|cc|}
\hline Item & Reliability if dropped \\
\hline traq1 & .89 \\
\hline traq2 & .89 \\
\hline traq3 & .90 \\
\hline traq4 & .88 \\
\hline traq5 & .91 \\
\hline traq6 & .90 \\
\hline traq7 & .90 \\
\hline traq8 & .89 \\
\hline traq9 & .89 \\
\hline traq10 & .90 \\
\hline
\end{tabular}

Results concerning internal consistency and reliability are presented in Table 1. Data shown than the TRAQ10 questionnaire carry high internal consistency and reliability even when an item is dropped.

The Cronbach's alpha was measured at . 9 [ $\left.\mathrm{Cl}_{95 \%}=.89-.92\right]$ for the full TRAQ10 questionnaire. When each of the TRAQ10 items was removed from the analysis in order to assess robustness, Cronbach's alpha remained high (varying from .89 to .90 with mean $_{\square}=.89, S D=.008$ ). All measures were above the minimum acceptable rate of .70 and was close from the maximum expected value of .9 .

\section{Confirmatory factor analysis}


Confirmatory factor analysis is presented in Figure 1. Results suggested a good model fit with the TRAQ10 questionnaire.

Significant items selected by the multiple regression were regrouped in three factors for three cognitive modules present in the ADHD. Items TRAQ2;4;9;10 were combined in the "Attention" factor, items TRAQ3;5;6;7 were combined in the "Inhibition/Impulsivity" factor, and items TRAQ1;8 were combined in the "Working memory" factor. Confirmatory factor analysis suggested that the 3-factors model fit well with the TRAQ10 questionnaire, except for the RMSEA which remains above the pre-defined cut-off $[\chi 2=74.86, p<.001, \mathrm{CFI}=.964, \mathrm{TLI}=.95, \mathrm{RMSEA}=.078, \mathrm{SRMR}=.044]$, even if some authors consider RMSEA $<.08$ as a good fit ${ }^{28}$.

Table 2

Standardized factor loadings of the 3-factor model of the TRAQ10.

\begin{tabular}{|llll|}
\hline Item & $\begin{array}{l}\text { Attention } \\
(\text { Mean=.82; SD=.1) }\end{array}$ & $\begin{array}{l}\text { Impulsivity } \\
(\text { Mean=.63; SD=.09) }\end{array}$ & $\begin{array}{l}\text { Working memory } \\
(\text { Mean=.79; SD=.002) }\end{array}$ \\
\hline traq4 & $0.924^{*}$ & - & - \\
\hline traq2 & $0.879^{*}$ & - & - \\
\hline traq9 & $0.811^{*}$ & - & - \\
\hline traq10 & $0.688^{*}$ & - & - \\
\hline traq3 & - & $0.693^{*}$ & - \\
\hline traq7 & - & $0.566^{\star}$ & - \\
\hline traq6 & - & $0.722^{\star}$ & - \\
\hline traq5 & - & $0.531^{\star}$ & - \\
\hline traq1 & - & - & $0.798^{\star}$ \\
\hline traq8 & - & - & $0.794^{\star}$ \\
\hline
\end{tabular}

Table 2 shown standardized factor loadings for the TRAQ10. Analysis revealed than all of the standardized factor loading ranged from .68 to .92 for attention, .53 to .69 for impulsivity, and was about to .79 for working memory module. All between-factors correlations were significant: correlation between inattention and impulsivity was $r=.76$ and correlation between inattention and working memory was $r=.88$. Finally, correlation between working memory and impulsivity was $r=.88$.

\section{Predictive power of the questionnaire}

Classification results are presented in Figure 2. The results showed that the algorithm was able to identify the diagnosis of ADHD in patients and exclude it in controls on the basis of TRAQ10 participants' responses with high accuracy. 
The GNB classifier which was trained on 132 randomly selected participants out of the 220 participants was able to identify the group appartenance of the remaining participant with an accuracy of .95 , identifying correctly 40 of the 42 ADHD participants and 44 of the 46 controls [sensitivity $=.95$; specificity $=.95 ; \mathrm{PPV}=.95 ; \mathrm{NPV}=.95$; Figure 2 . Left]. Further 10 -fold cross validation procedure shown a mean accuracy of .88 (SD $=.09$; Figure 2 . Right).

\section{Discussion}

The present study verified the reliability and the construct validity of the Trognon \& Richard ADHD Questionnaire 10-items French version for Adults (TRAQ10-AF).

Cronbach's alpha values suggested that the TRAQ10 was internally reliable, with measured alpha

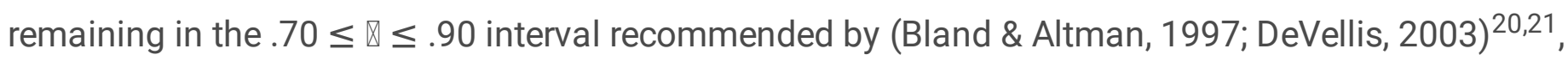
except when the item 5 is dropped, and where the measured alpha exceeds the upper limit of .01 .

Further confirmatory factor analysis supported our three-factor structure of the TRAQ10 questionnaire, with items TRAQ2;4;9;10 grouped in the "Attention" factor, TRAQ3;5;6;7 grouped in the "Inhibition/Impulsivity" factor, and TRAQ1;8 grouped in the "Working memory" factor. Analysis showed that this model met the standards defined by (Hu \& Bentler, 1999) ${ }^{26}$, except for the RMSEA which remains $>.06$, even if its value which was measured $<.08$ is acceptable in regard of (Awang, 2012) ${ }^{28}$ guidelines. Furthermore, all of items highly loaded on their attributed factors, and all factors correlated with the others. However, these factorial measures had to be replicated in neuropsychological studies and evaluated in their relatedness to cognitive impairments.

We then performed a classification using machine-learning approaches and shown that TRAQ10 questionnaire could be used in order to identify ADHD diagnosis with high accuracy and predictive values.

\section{Conclusion}

We concluded from these analyses that TRAQ10 can be used as a diagnostic tool to highlight ADHD in french populations. From a clinical point of view, in order to significantly reduce the time required to carry out the multidisciplinary diagnosis of ADHD, we believe that this scale could be used during a first consultation or at the end of the consultation to support the health professional's clinical expertise and serve as a decision aid in deciding whether or not to pursue a multidisciplinary diagnostic process.

\section{Declarations}

\section{Ethics approval and consent to participate}

Consent was obtained online before the completion of the survey using four questions in the questionnaire. 
- I am voluntarily participating in this research

- I can stop my participation at any time without having to give explanations

- All information I provide will be kept confidential and my identity will never be disclosed.

- I authorize the conservation and use of this confidential data for scientific research in psychology.

The study did not collect any identifiable data (no name, date of birth, location, or IP address), and only collected the time stamp of the pass (format: DD/MM/YY H:M:S). All participants received detailed information regarding the purpose of the study and provided online informed consent to participate in the study. The survey was anonymous to ensure the confidentiality and reliability of the data. All procedures were conducted in accordance with the principles of the Declaration of Helsinki and the study protocol was approved by the Institutional Review Board Commission Nationale de I'Informatique et des Libertés (CNIL - registration 2224719v0).

Consent for publication

Not applicable.

Availability of data and materials

The dataset used during the study is available in (Supplementary File 1)

Competing interests

Not applicable.

Funding

Not applicable

Author's contributions:

AT and MR designed the scale. MR collected the data, and AT performed all analysis.

Acknowledgements

Not applicable.

\section{References}

1. Scassellati, C., Bonvicini, C., Faraone, S. V. \& Gennarelli, M. Biomarkers and attentiondeficit/hyperactivity disorder: a systematic review and meta-analyses. J Am Acad Child Adolesc Psychiatry 51, 1003-1019.e20 (2012).

2. Biederman, J. \& Faraone, S. V. Attention-deficit hyperactivity disorder. Lancet 366, 237-248 (2005). 
3. Faraone, S. V. et al. Attention-deficit/hyperactivity disorder in adults: An overview. Biological Psychiatry 48, 9-20 (2000).

4. Kooij, S. J. et al. European consensus statement on diagnosis and treatment of adult ADHD: The European Network Adult ADHD. BMC Psychiatry 10, 67 (2010).

5. Faraone, S. V. et al. Attention-deficit/hyperactivity disorder. Nature Reviews Disease Primers 1,1-23 (2015).

6. Turning Attention to ADHD | Express Scripts. https://www.express-scripts.com/corporate/drug-trendreport/turning-attention-adhd.

7. Stockl, K. M., Hughes, T. E., Jarrar, M. A., Secnik, K. \& Perwien, A. R. Physician perceptions of the use of medications for attention deficit hyperactivity disorder. J Manag Care Pharm 9, 416-423 (2003).

8. American Psychiatric Association. Diagnostic and statistical manual of mental disorders (5th ed.). (2013).

9. Foreman, D. M., Foreman, D., Prendergast, M. \& Minty, B. Is clinic prevalence of ICD-10 hyperkinesis underestimated? Impact of increasing awareness by a questionnaire screen in an UK clinic. Eur Child Adolesc Psychiatry 10, 130-134 (2001).

10. Dalsgaard, S., Leckman, J. F., Mortensen, P. B., Nielsen, H. S. \& Simonsen, M. Effect of drugs on the risk of injuries in children with attention deficit hyperactivity disorder: a prospective cohort study. The Lancet Psychiatry 2, 702-709 (2015).

11. Chang, W. C. et al. Clinical and cognitive predictors of vocational outcome in first-episode schizophrenia: a prospective 3 year follow-up study. Psychiatry Res 220, 834-839 (2014).

12. Dalsgaard, S., Mortensen, P. B., Frydenberg, M. \& Thomsen, P. H. ADHD, stimulant treatment in childhood and subsequent substance abuse in adulthood - a naturalistic long-term follow-up study. Addict Behav 39, 325-328 (2014).

13. Barkley, R. A., Murphy, K. R. \& Fischer, M. ADHD in Adults: What the Science Says. (The Guilford Press, 2010).

14. Furczyk, K. \& Thome, J. Adult ADHD and suicide. ADHD Atten Def Hyp Disord 6, 153-158 (2014).

15. van de Glind, G. et al. Validity of the Adult ADHD Self-Report Scale (ASRS) as a screener for adult ADHD in treatment seeking substance use disorder patients. Drug and Alcohol Dependence 132, 587-596 (2013).

16. Ustun, B. et al. The World Health Organization Adult Attention-Deficit/Hyperactivity Disorder SelfReport Screening Scale for DSM-5. JAMA Psychiatry 74, 520-526 (2017).

17. Lozano, L. M., García-Cueto, E. \& Muñiz, J. Effect of the number of response categories on the reliability and validity of rating scales. Methodology: European Journal of Research Methods for the Behavioral and Social Sciences 4, 73-79 (2008).

18. Weijters, B., Cabooter, E. \& Schillewaert, N. The effect of rating scale format on response styles: The number of response categories and response category labels. International Journal of Research in Marketing 27, 236-247 (2010). 
19. Butzbach, M. et al. Basic processes as foundations of cognitive impairment in adult ADHD. J Neural Transm 126, 1347-1362 (2019).

20. Bland, J. M. \& Altman, D. G. Cronbach's alpha. BMJ 314, 572 (1997).

21. DeVellis, R. F. Scale Development: Theory and Applications. (SAGE, 2003).

22. Bentler, P. M. Comparative fit indexes in structural models. Psychol Bull 107, 238-246 (1990).

23. Tucker, L. R. \& Lewis, C. A reliability coefficient for maximum likelihood factor analysis. Psychometrika 38, 1-10 (1973).

24. Bentler, P. M. EQS: structural equations program manual. undefined https://www.semanticscholar.org/paper/EQS-\%3A-structural-equations-program-manualBentler/3b39d1d27934a461f04e0e076ddb6da5b87193b0 (1989).

25. Steiger, J. H. Statistically based tests for the number of common factors. (1980) doi:null.

26. Hu, L. \& Bentler, P. M. Cutoff criteria for fit indexes in covariance structure analysis: Conventional criteria versus new alternatives. Structural Equation Modeling: A Multidisciplinary Journal 6, 1-55 (1999).

27. Zhang, $\mathrm{H}$. The Optimality of Naïve Bayes. in In FLAIRS2004 conference(2004).

28. Awang, Z. Structural equation modeling using AMOS graphic. (Penerbit Universiti Teknologi MARA, 2012).

\section{Figures}




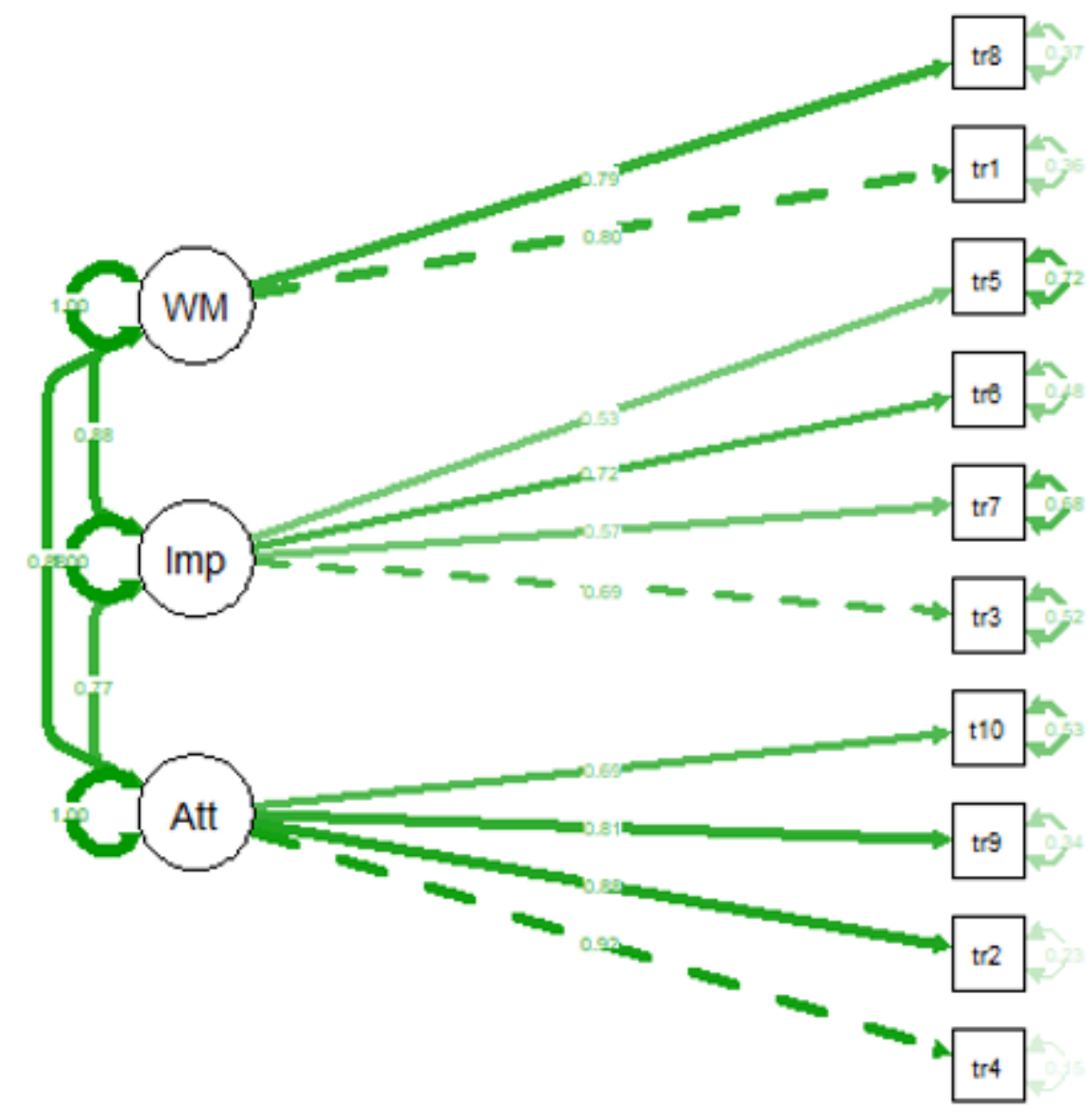

\section{Figure 1}

Model fit for the 3-factor model of the TRAQ10 questionnaire [Att : Attention ; Imp : Impulsivity ; WM : Working Memory]
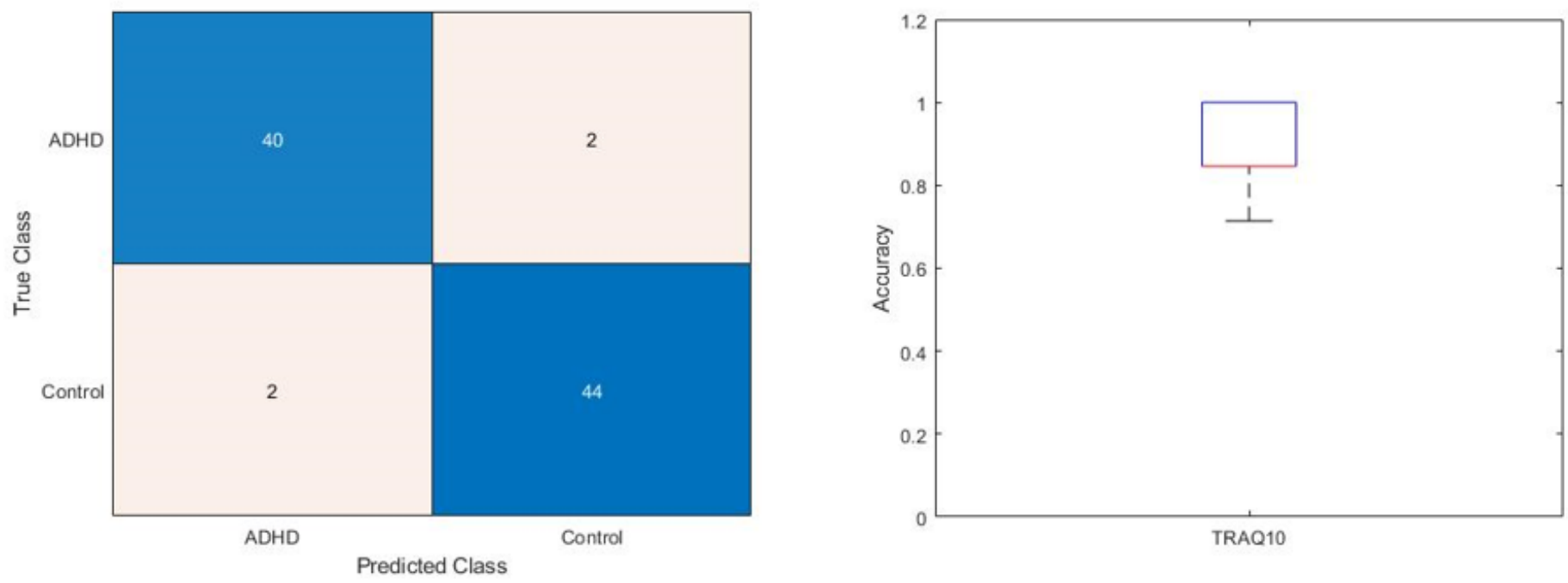
Figure 2

Classification results of the TRAQ10 questionnaire. [Left : Confusion matrix of the GNB Classifier ; Right : Mean accuracy of the 10 -fold cross-validation.]

\section{Supplementary Files}

This is a list of supplementary files associated with this preprint. Click to download.

- supplementaryFile1.xlsx 